\title{
Investigation of Changes in the Electrical Properties of Novel Knitted Conductive Textiles During Cyclic Loading
}

\author{
Cristina Isaia, Donal McNally, Simon A. McMaster, David T. Branson
}

\begin{abstract}
Combining stainless steel with polyester fibres adds an attractive conductive behaviour to the yarn. Once knitted in such a manner, fabrics develop sensing properties that make the textiles, also known as e-textiles, suitable for smart/wearable applications. Structural deformations of the fibres (e.g. stretching) will cause changes in the conductivity of the fabric. This work investigates changes in the electrical properties exhibited by four knitted conductive textiles made of $\mathbf{2 0 \%}$ stainless steel and $\mathbf{8 0 \%}$ polyester fibres during cyclic loading. The samples were preconditioned first with $\mathbf{5 0 0}$ hundred cycles of unidirectional elongation and, after a rest interval, tested again for repeatability at the same conditions. In both cases the electrical behaviour stabilises after a few tens of cycles. In particular the repeatability test exhibited a considerably smaller settling time and a larger resistance due to the mechanical stabilisation and the loosening of the fabrics, respectively. It was found that the current provided to the fabrics affects the resistance measurements by decreasing the resistance value at which the samples become electrically stable. The reported findings present a valid method for the electrical characterisation of conductive textiles for use in further studies as a wearable technology.
\end{abstract}

\section{INTRODUCTION}

Wearable electronic textile, also known as e-textile, varies from electronic components attached superficially to the garment to yarns that have been treated or modified to behave as smart fabric sensors. Several authors have described the methods by which fibres can develop sensing properties by coating a thin layer of piezoresistive material on conventional fabrics [1], knitting conductive yarns with nonconductive yarns [2] or by stitches [3], or attachment to the top of the fabric [4].

To improve the usability and performance of the wearable sensor it is essential to investigate the fabrics' behaviour while deformation is occurring (e.g. stretching). For instance, when a load is applied to the electrically conductive textile a change of the fabric electric resistance results from structural deformations. To determine the overall equivalent resistance of a knitted conductive yarn two types of electrical resistance might be taken into account: the length-related resistance $R_{l}$ and the contact resistance $R_{c}$ [5] described according to Ohm's law [6] and Holm's contact theory [7], respectively. Many papers have described theoretical and experimental investigations of the relationship between the resistance and

Cristina Isaia is with the Faculty of Engineering, University of Nottingham, United Kingdom (e-mail: eaxci@nottingham.ac.uk).

Donal McNally is with the Faculty of Engineering, University of Nottingham, United Kingdom (e-mail: donal.monally@nottingham.ac.uk).

Simon A. McMaster is with the Footfalls \& Heartbeats (UK) Limited, Grantham, United Kingdom (e-mail: simon@footfallsandheatbeats.com).

David T. Branson is with the Faculty of Engineering, University of Nottingham, United Kingdom (e-mail: david.branson@nottingham.ac.uk). the elongation of conductive fabrics. Electromechanical properties of knitted wearable sensors have been studied using the hexagon resistance model [8] and the conductive deformation behaviour of the fabric has been predicted and experimentally verified [9]. Moreover, the elongation and time dependent behaviour of a stretched fabric [10] have also been taken into account, as well as the temperature effect on the conductivity of the knitted fabrics [11].

Nonetheless, few studies have fully verified the influence of the mechanical properties over the electrical behaviour of the fabrics. In particular there is no report of the electrical behaviour of the conductive textiles during preconditioning and of the effects of the current on the fabric resistance variation.

The use of conductive fabrics as position and motion sensors requires repeatability of the electrical properties. As the overall electrical resistance is related to the yarn length and contact force between yarns, it is not comprehensive to theoretically study the electrical properties without taking into account the effect of the underlying mechanical features. Repeatability can be strongly influenced by the mechanical properties of the interlocked fibres and/or the supporting garment in which the fabrics are embedded.

The objectives of this study are to investigate the role of mechanical preconditioning on the electrical behaviour of knitted conductive fabrics. In particular it was studied whether the electrical properties shown by the textiles change during cyclic mechanical loading, after a rest period and if they are affected by the way the fabrics are mechanically loaded or electrically driven.

According to the electrical performance exhibited by the presented conductive fabrics, textile-based wearable sensors may be developed for rehabilitative and biomechanics purposes. The challenge in future will be to study the usability of the described conductive fabrics as less invasive sensors embedded in leggings to control a prosthetic knee.

\section{EXPERIMENTAL SET UP}

\section{A. Materials}

Four conductive knitted samples with different stitch patterns, Single Jersey, SP27, SP29 and SP31 (C) Footfalls \& Heartbeats (UK) Limited) were employed for this investigation. Single Jersey (SJ) is a control stitch structure composed of a series of interconnecting loops creating $100 \%$ of the sensor area. SP27, SP29 and SP31 consist of a minimum of $50 \% \mathrm{SJ}$ and a combination of miss or tuck stitches that constitutes the balance percentage. The samples were formed by a 'course' of knit, which means that a horizontal row of needle loops was produced by adjacent needles during the same knitting cycle. Each sample was 250 $\mathrm{mm} \times 50 \mathrm{~mm}$ (height $\mathrm{x}$ width) and made of both conductive $(150 \mathrm{~mm}$ x $50 \mathrm{~mm})$ and non-conductive yarns. The 
conductive yarn was a spun staple fibre composed of $20 \%$ stainless steel and $80 \%$ polyester (PES) stable fibres $(80 / 20$ polyester/INOX ${ }^{\circledR}$ ), made by Schoeller, Bregenz (yarn count $\mathrm{Nm} \mathrm{50/2}$ which means 2 yarns, each of $50 \mathrm{~m}$, weigh $2 \mathrm{~g}$ ). The non-conductive yarn was a textured polyester multifilament yarn (yarn count $150 \mathrm{~d} / 48 \mathrm{f}$ ), whose mass density of fibres was 150 denier (mass in grams per 9000 meters) and the number of filament that were spun together in the yarn was 48 , as shown in Fig. 1 a. The samples were knitted on a flatbed Shima Seiki 122S 10 gauge weft knitting machine. An example of the weft knitting process is illustrated in Fig. $1 \mathrm{~b}$ (Single Jersey structure).

\section{B. The equipment}

The tensile machine used for investigating the conductive fabrics' behaviour during cyclic loading was an Instron 8801 Fatigue machine (Fig. 1 c). The specimens were gripped between polytetrafluoroethylene (PTFE) insulating pads to avoid any contact between the conductive area of the samples and the metallic grips of the tensile machine. To electrically characterise the conductive samples, a four-wire resistance measurement was chosen and different constant current values provided to the specimens by a purposely manufactured electronic circuitry. Data were then collected with a NI USB-6003 data acquisition system at a $1000 \mathrm{~Hz}$ sampling rate.
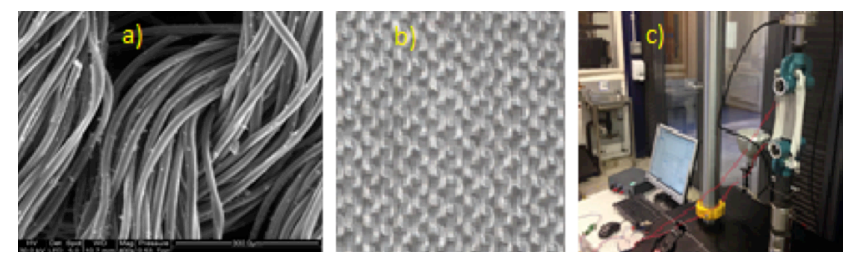

Figure 1. a) ESEM image of PPY coated 150/48 dtex textured polyester (C) Footfalls \& Heartbeats (UK) Limited) b) Single Jersey structure as a result of the weft knitting process c) The equipment used in the study.

\section{METHOD}

To investigate the electrical properties two tests sessions of five hundred stretch-recovery cycles were conducted for each fabric at three constant currents (1mA, 3mA and $6 \mathrm{~mA}$ ) and three elongation rates $(9 \mathrm{~mm} / \mathrm{s}, 12 \mathrm{~mm} / \mathrm{s}$ and $15 \mathrm{~mm} / \mathrm{s})$. The specimens, whose original length between the clamps was $100 \mathrm{~mm}$, were elongated in their longitudinal direction with a triangle wave of $20 \mathrm{~mm}$ amplitude and $0.22,0.3$ and $0.375 \mathrm{~Hz}$ frequencies corresponding to the three elongation rates selected for the tests.

\section{A. Preconditioning of the test specimens}

The first test session was necessary for the mechanical preconditioning of the fabrics and for studying whether the electrical properties of the specimens stabilise after a number of cycles.

\section{B. Repeatability test}

To find out whether a rest period would reset the preconditioning effects, a second repeatability session followed after 5 minutes rest interval without modifying the setting of the specimens in the clamps. This choice of time was made to electrically characterise each sample in a way compatible as possible with a real application (e.g. the fabric might be sewn in a garment at the knee and used again after a limited sitting/rest period).

\section{Post processing procedure}

Data collected with the acquisition system were then post processed in Matlab. To reduce the electrical noise superimposed on the total resistance measured during deformation a Savitzky-Golay filter of polynomial order $N=3$ was applied to the signal (Fig. 2). This window length was chosen to accurately follow the fabric resistance variation and filter out fast changes in the signal due to the noise, without distortions/delays being added to the results.

Next, a peak analysis was applied to the filtered signal to detect the maximum and minimum resistance values and a Savitzky-Golay filter was used again to reduce the variability in the extracted data points. Subsequently, interpolation curves passing through the maximum and minimum filtered values were established to compare in the time domain the data points as they were separated from each other by half a period (Fig. 3).

One of the main features for describing the electrical behaviour of the samples, independent of the fabric design and composition, was the peak-to-peak span. It was determined by subtracting the interpolated filtered minimum curve from the interpolated filtered maximum one. Moreover, by calculating the derivatives of these curves (Fig. 4) it was possible to study how the signal varied with the time. This method was useful for finding out the settling time of all samples.

Another feature describing the fabrics' electrical properties was the mean of the filtered resistance calculated from the settling time onwards. It will be referred to as stabilised average resistance in the rest of the paper.

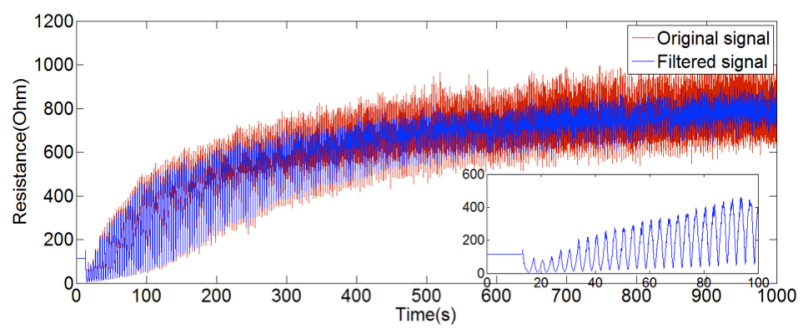

Figure 2. a) Example of filtering the electrical resistance for the SP29 sample sourced with $3 \mathrm{~mA}$ and tested at $12 \mathrm{~mm} / \mathrm{s}$ during preconditioning session b) Zoom of the first 25 elongations.

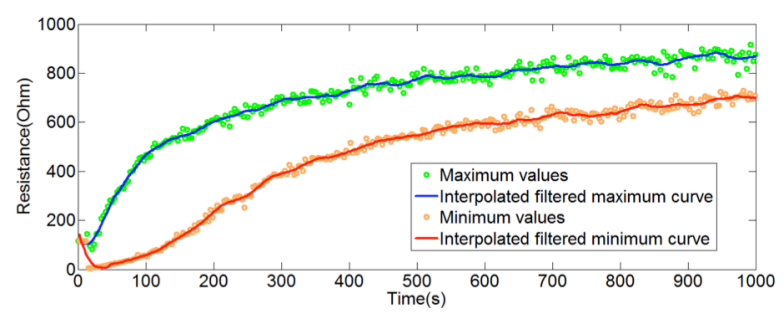

Figure 3. Interpolated filtered maximum and minimum curves for the SP29 sample sourced with $3 \mathrm{~mA}$ and tested at $12 \mathrm{~mm} / \mathrm{s}$ during preconditioning test. 


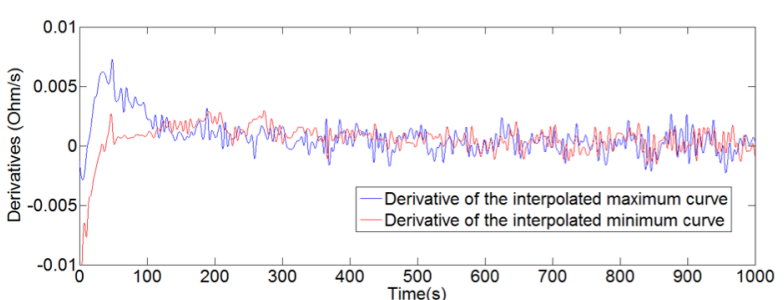

Figure 4. Derivatives of the interpolated maximum and minimum curves for the SP29 sample sourced with $3 \mathrm{~mA}$ and tested at $12 \mathrm{~mm} / \mathrm{s}$ during preconditioning test.

\section{RESULTS AND DISCUSSION}

\section{A. Preconditioning of test specimens}

The effect of the preconditioning test phase is the stabilisation of the electrical properties within the samples after a certain number of cycles. It was found that after an initial maximum in the peak-to-peak span value, corresponding to the initial cycles in which the electrical equilibrium is not reached, the span settled to a constant value. This phenomenon is illustrated in Fig. 5, referring for simplicity to the SP29 sample sourced with $3 \mathrm{~mA}$ and tested at $12 \mathrm{~mm} / \mathrm{s}$, and reported numerically in Table II. Table I reports the number of cycles for the stabilisation of the electrical properties. Both tables' results are obtained by taking the mean of all samples' values at three different currents and elongation rates.

\section{B. Repeatability test}

During the repeatability tests the rest period between the first and second session does not cancel the effect of the preconditioning process. In fact, it was demonstrated that the samples show again a stabilisation of the peak-to-peak span and, compared to the preconditioning session, it occurs in a shorter period of time (Fig. 5) and with a slight variation (Table II). This tendency is common for all samples at any current and elongation rate.

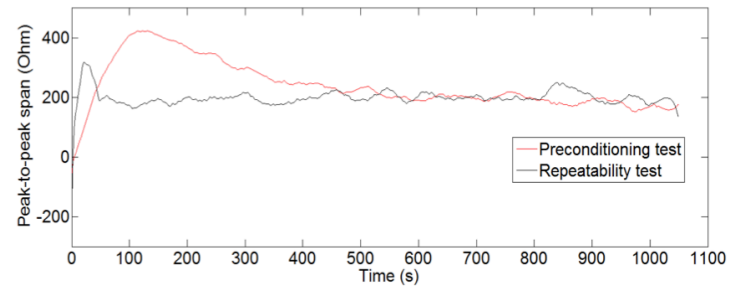

Figure 5. Comparison of the peak-to-peak span during preconditioning and repeatability tests for the SP29 sample sourced with $3 \mathrm{~mA}$ and tested at $12 \mathrm{~mm} / \mathrm{s}$.

In addition, the stabilised average resistance increased from the preconditioning to repeatability tests, as reported in Table II. This is likely due to an increased total resistance between the trials as a consequence of the mechanical stresses and deformations the samples were subject to resulting in:

- Decreased contact pressure causing an increase in the contact resistance. This can be explained by observing that between the trials the fabric becomes looser.
- Increased yarn length and/or decreased yarn cross sectional area resulting in an increase of the length-related resistance. This can also be explained by the loosening of the fabric.

TABLE I. NUMBER OF CYCLES FOR ELECTRICAL STABILISATION

\begin{tabular}{|c|c|c|c|c|}
\hline Samples & Test & Mean & $\begin{array}{l}\text { Standard } \\
\text { deviation }\end{array}$ & Decrement \\
\hline \multirow{2}{*}{$\begin{array}{l}\text { Single } \\
\text { Jersey }\end{array}$} & Preconditioning & 38 & 13 & \multirow{2}{*}{$53 \%$} \\
\hline & Repeatability & 18 & 9 & \\
\hline \multirow{2}{*}{ SP27 } & Preconditioning & 45 & 29 & \multirow{2}{*}{$69 \%$} \\
\hline & Repeatability & 14 & 1 & \\
\hline \multirow{2}{*}{ SP29 } & Preconditioning & 51 & 35 & \multirow{2}{*}{$68 \%$} \\
\hline & Repeatability & 16 & 8 & \\
\hline \multirow{2}{*}{ SP31 } & Preconditioning & 46 & 13 & \multirow{2}{*}{$69 \%$} \\
\hline & Repeatability & 14 & 3 & \\
\hline
\end{tabular}

TABLE II. STABILISED AVERAGE RESISTANCE AND STABILISED PEAKTO-PEAK SPAN

\begin{tabular}{|c|c|c|c|c|c|}
\hline Sample & Test & 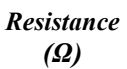 & $\begin{array}{l}\text { Resistance } \\
\text { Increment }\end{array}$ & $\begin{array}{c}\text { Span } \\
(\Omega)\end{array}$ & $\begin{array}{c}\text { Span } \\
\text { Variation }\end{array}$ \\
\hline \multirow{2}{*}{$\begin{array}{l}\text { Single } \\
\text { Jersey }\end{array}$} & Preconditioning & 1448.5 & \multirow{2}{*}{$17 \%$} & 409.9 & \multirow{2}{*}{$+9 \%$} \\
\hline & Repeatability & 1696 & & 447.2 & \\
\hline \multirow{2}{*}{ SP27 } & Preconditioning & 943.6 & \multirow[b]{2}{*}{$51 \%$} & 357.2 & \multirow[b]{2}{*}{$+13 \%$} \\
\hline & Repeatability & 1424.5 & & 403.1 & \\
\hline \multirow[b]{2}{*}{ SP29 } & Preconditioning & 782.2 & \multirow[b]{2}{*}{$45 \%$} & 322.3 & \multirow[b]{2}{*}{$-2 \%$} \\
\hline & Repeatability & 1136.6 & & 315.8 & \\
\hline \multirow{2}{*}{ SP31 } & Preconditioning & 865.2 & \multirow{2}{*}{$34 \%$} & 326.5 & \multirow[b]{2}{*}{$+5 \%$} \\
\hline & Repeatability & 1159.7 & & 342.1 & \\
\hline
\end{tabular}

\section{Effect of the current on resistance measurement}

It was further found that the electrical properties of the conductive samples change with the provided current. Firstly, the stabilised average resistance decreases for increasing currents during both preconditioning and repeatability tests. For instance, increasing the current from $1 \mathrm{~mA}$ to $3 \mathrm{~mA}$ and from $3 \mathrm{~mA}$ to $6 \mathrm{~mA}$ resulted in a decrease in the stabilised average resistance of $60 \%$ and $39 \%, 57 \%$ and $46 \%, 53 \%$ and $46 \%$ for samples undergoing elongation rates of 9,12 and $15 \mathrm{~mm} / \mathrm{s}$, respectively. An explanation of this phenomenon is the Ohmic contact between the stainless fibres due to the $\mathrm{p}$ type semiconducting behaviour of the oxidation layer on the surface of the fibres [12]. For simplicity, Fig. 6 shows the described phenomenon only for samples tested at $12 \mathrm{~mm} / \mathrm{s}$ during repeatability tests.

Secondly, the current sourced to the samples has also an effect on the stabilisation of the peak-to-peak span during both preconditioning and repeatability tests. As shown in Fig. 
7, referring to the preconditioning test, the values at which the peak-to-peak span settles are mainly dependent on the provided current and not significantly on the elongation rate.

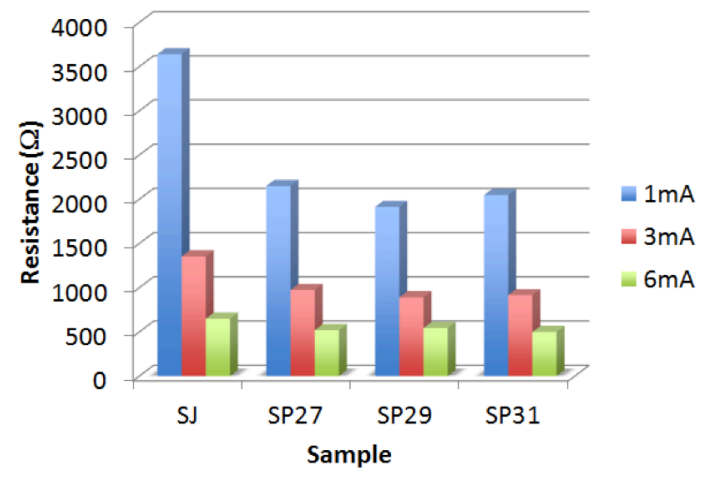

Figure 6. Stabilised average resistance for all samples tested at $12 \mathrm{~mm} / \mathrm{s}$ during repeatability test.

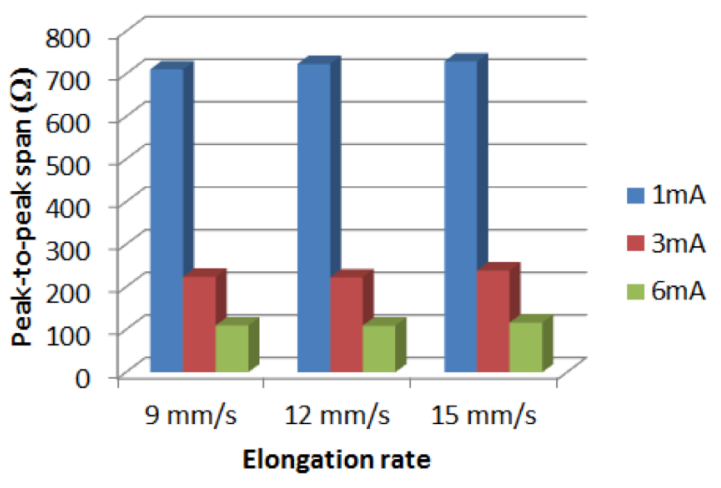

Figure 7. Peak-to-peak span settling values for all samples during preconditioning test at different currents and elongation rates.

\section{CONCLUSION}

This paper has investigated the effect of mechanical preconditioning tests on the electrical properties of novel textiles knitted with conductive yarns. It was discovered that, after an initial preconditioning round of testing, the stabilisation of the electrical behaviour was faster (i.e. took $65 \%$ fewer cycles on average) and more repeatable (mean variation of $6.25 \%$ ). In particular the peak-to-peak span, which corresponds to the sensitivity of the transducer, was found to stabilise rapidly and that this stabilised value was conserved after a period of rest and further cyclic loading. These findings show the ability of the tested sensors to produce similar output in repeated tests under the same measurement conditions. Moreover, during repeatability tests it was also observed a phenomenon of decreasing resistance with increasing current (i.e. on average $57 \%$ decreased resistance from $1 \mathrm{~mA}$ to $3 \mathrm{~mA}$ and $43 \%$ from $3 \mathrm{~mA}$ to $6 \mathrm{~mA}$ ), proving the Ohmic behaviour of the contact between fibres.

Results from this work will be used in further studies investigating the use of similar fabric sensors in clothing (e.g. leggings) to study human motion capture. For this purpose, the peak-to-peak span repeatability will be a key feature in assessing the validity of specific conductive fabrics as sensors and in establishing which stitch pattern and composition guarantee variability smaller than predetermined acceptance criteria.

\section{ACKNOWLEDGMENT}

We would like to thank the Footfalls and Heartbeats for providing the Single Jersey, SP27, SP29 and SP31 knitted conductive fabrics (C) Footfalls \& Heartbeats (UK) Limited) and the School of Materials Textile Laboratory at the University of Manchester for manufacturing the samples. We are also grateful to Dr. De Focatiis from the University of Nottingham for providing assistance during testing and the polytetrafluoroethylene insulating pads.

\section{REFERENCES}

[1] D. De Rossi, F. Carpi, F. Lorussi, A. Mazzoldi, E. Scilingo and A. Tognetti, "Electroactive fabrics for distributed, conformable and interactive systems", in Sensors, Proceedings of IEEE, Vol. 2, 2002, pp. 1608-1613.

[2] M. Pacelli, L. Caldani and R. Paradiso, "Textile piezoresistive sensors for biomechanical variables monitoring", in Engineering in Medicine and Biology Society, EMBS'06. 28th Annual International Conference of the IEEE, 2006, pp. 5358-5361.

[3] G. Gioberto and L. Dunne, "Theory and characterization of a topthread coverstitched stretch sensor", in Systems, Man, and Cybernetics (SMC), 2012 IEEE International Conference on, 2012, pp. 32753280.

[4] C. Mattmann, F. Clemens and G. Tröster, "Sensor for measuring strain in textile", Sensors 2008, Vol. 8, no. 6, pp. 3719-3732.

[5] H. Zhang, X. M. Tao, S. Y. Wang and T. X. Yu, "Electro-Mechanical properties of knitted fabric made from conductive multi-filament yarn under unidirectional extension", Textile Research Journal, 2005, Vol. 75 , no. 8, pp. 598-606.

[6] M.S. Gupta, "George Simon Ohm and Ohm's Law", Education, IEEE Transaction on, 1980, Vol. 23, no. 3.

[7] R. Holm and E. Holm, "Electric Contact Theory and Application", 4th Ed., New York: Springer-Verlag, 1967.

[8] J. Wang, H. Long, S. Soltanian, P. Servati and F. Ko, "Electromechanical properties of knitted wearable sensors: part Itheory", Textile Research Journal, 2014, Vol. 84, no. 1, pp. 3-15.

[9] J. Wang, H. Long, S. Soltanian, P. Servati and F. Ko, "Electromechanical properties of knitted wearable sensors: part 2Parametric study and experimental verification", Textile Research Journal, 2014, Vol. 84, no. 2, pp. 200-213.

[10] A. Ehrmann, F. Heimlich, A. Brücken, M. O. Weber and R. Haug, "Suitability of knitted fabrics as elongation sensors subject to structure, stitch dimension and elongation direction", Textile Research Journal, 2014, Vol. 84, no. 18, pp. 2006-2012.

[11] J. Tong, X. Tao, W. M. Au and L. Li, "Temperature effect on the conductivity of knitted fabrics embedded with conducting yarns", Textile Research Journal, 2014, Vol. 84, no.17, pp. 1849-1857.

[12] J. Yin, C. D’Haese and B. Nysten, "Surface electrical properties of stainless steel fibres: An AFM-based study", Applied Surface Science, Elsevier, 2015, Vol. 330, pp. 65-73. 